where $A, B$ are arbitrary real constants, and $\alpha$ is an arbitrary complex number. It is readily confirmed by direct computation that the constants $A, B$, and $\alpha$ give rise to an arbitrary, uniform hydrostatic stress-field in the image domain $R^{*}$. The constant $\kappa$, on the other hand, affects merely the scale of the image stress-distribution. We may therefore put $A=B=\alpha=0, \kappa=1$. This completes the proof.

\title{
REFERENCES
}

1. J. H. Michell, The inversion of plane stress, Proc. London. Math. Soc. 34, 134 (1901).

2. Ludwig Foeppl, Drang und Zwang, vol. 3, Leibniz Verlag, Muenchen, 1947.

3. C. Weber, Spannungsverteilung in Blechen mit mehreren kreisrunden Loechern, ZS. angew. Math. Mech. 2, 267 (1922).

4. W. Olszak, Beitraege zur Anwendung der Inversionsmethode bei Behandlung von ebenen Problemen der Elastizitaetstheorie, Ingenieur Archiv 6, 402 (1935).

5. R. D. Mindlin, Stress systems in a circular disk under radial forces, J. Appl. Mech. 4, 115 (1937).

6. V. P. Jensen and D. L. Holl, An application of derivatives of non-analytic functions in plane stress problems, Bull. Amer. Math. Soc. 43, 256 (1937).

7. Edward Kasner, A new theory of polygenic functions, Science 66, 581 (1927).

8. E. R. Hedrick, Non-analytic functions of a complex variable, Bull. Amer. Math. Soc. 39, 75 (1933).

9. Rufus Isaacs, Planar elasticity as a potential theory, Mathematicas y Fisca Theorica, Universidad Nacional de Tucuman, (A) 6, 263 (1948).

\section{A MINIMUM PRINCIPLE FOR STRUCTURAL STABILITY*}

By H. J. WEISS AND G. H. HANDELMAN (Carnegie Institute of Technology)

1. Statement of the problem. In a recent paper, ${ }^{1} \mathrm{~W}$. Prager has discussed the problem of structural stability from the following point of view. Consider a given configuration of a deformable body, referred to a fixed system of rectangular axes, $x_{i}(i=1,2,3)$, under a set of stresses $\lambda \sigma_{i j}$ which are in equilibrium with given surface tractions. These stresses are prescribed only to within the arbitrary constant factor $\lambda$. The configuration is assumed to be stable if $\lambda$ is sufficiently small. A system of infinitesimal perturbation displacements $u_{i}$ is then applied, and the question is asked for what values of the factor $\lambda$ will the equilibrium become indifferent.

The solution to the problem leads to the following system of linear, homogeneous, second order, partial differential equations

$$
J_{i, i}=0,
$$

subject to the homogeneous boundary conditions on the surface

$$
J_{i j} n_{i}=0,
$$

where

$$
J_{i i}=\left[\tau_{i j}+\frac{1}{2} \lambda\left(\sigma_{i p} \epsilon_{p i}-\sigma_{i p} \epsilon_{p i}\right)-\lambda \sigma_{i p} \omega_{p i}\right]
$$

*Received May 29, 1950.

'W. Prager, The general variational principle of the theory of structural stability, Q. Appl. Math., 4, 378-384 (1947). 
The quantities appearing in the definition of $J_{i i}$ are defined below: the strain, $\epsilon_{i i}$, associated with the perturbations $u_{i}$ is given by

$$
\epsilon_{i j}=\frac{1}{2}\left(u_{i, i}+u_{i, i}\right) \text {; }
$$

the additional stresses produced by these strains satisfy the linear relation

$$
\tau_{i j}=C_{i j k l} \epsilon_{k l}
$$

in which $C_{i j k l}$ is symmetric with respect to $i$ and $j$ and with respect to $k$ and $l ; \omega_{i i}$ is the local rotation due to the displacement $u_{i}$,

$$
\omega_{i j}=\frac{1}{2}\left(u_{i, i}-u_{i, i}\right) ;
$$

and $n_{i}$ is the unit vector normal to the original equilibrium surface. The problem discussed here will be restricted to the elastic case; thus

$$
C_{i j k l}=2 G_{0}\left(\delta_{i k} \delta_{j l}-\frac{\nu}{1-2 \nu} \delta_{i j} \delta_{k l}\right),
$$

where $G_{0}$ is the modulus of rigidity and $\nu$ is Poisson's ratio. It should be noted that $C_{i j k l}$ is symmetric with respect to $(i j)$ and $(k l)$. The initial stresses $\sigma_{i j}$ are symmetric with respect to $i$ and $j$ and satisfy the equilibrium equations

$$
\sigma_{i j, i}=0 .
$$

Prager has shown that the differential equations (1), subject to boundary conditions (2), are equivalent to the statement that the first variation of the integral

$$
\int_{V}\left[C_{i j p q} \boldsymbol{\epsilon}_{i j} \boldsymbol{\epsilon}_{p q}+\lambda \sigma_{i j}\left(u_{p, i} u_{p, i}-\epsilon_{p i} \epsilon_{p j}\right)\right] d V
$$

vanishes. Integration is carried out over the volume $\mathrm{V}$ of the original unperturbed body and only the displacements $u_{p}$ are varied.

2. The minimum principle. It will be shown that the eigenvalues $\lambda$ and the corresponding eigenfunctions of Eqs. (1) may be obtained from a minimum principle applied to an integral closely associated with that of Eq. (4). Since the problem in question is one of stability, only the first eigenvalue will be considered.

It is more convenient to express Eqs. (1) and (2) in terms of the displacement $u_{i}$ directly. Thus, from Eqs. (3a), (3b), and (3c), $J_{i i}$ may be written as

$$
J_{i i}=C_{i j p q} u_{p, Q}+\frac{\lambda}{4}\left[\sigma_{p i}\left(3 u_{i, p}-u_{p, i}\right)-\sigma_{p i}\left(u_{i, p}+u_{p, i}\right)\right] ;
$$

and the integral (4) becomes

If

$$
\int_{V}\left[C_{i j p q} u_{i, i} u_{p, q}+\frac{\lambda}{4} \sigma_{i j}\left(3 u_{p, i} u_{p, i}-u_{i, p} u_{i, p}-u_{p, i} u_{i, p}-u_{i, p} u_{p, j}\right)\right] d V .
$$

$$
\begin{aligned}
D(u) & =\int_{V} C_{i j p q} u_{i ; j} u_{p, q} d V \\
D(u, \nu) & =\int_{V} C_{i, p a} u_{i, j} v_{p, o} d V ;
\end{aligned}
$$




$$
\begin{gathered}
H(u)=-\frac{1}{4} \int_{V} \sigma_{i j}\left(3 u_{p, i} u_{p, i}-u_{i, p} u_{i, p}-u_{p, i} u_{i, p}-u_{i, p} u_{p, i}\right) d V, \\
H(u, v)=-\frac{1}{4} \int_{V} \sigma_{i j}\left(3 u_{p, i} v_{p, i}-u_{i, p} v_{i, p}-u_{p, i} v_{i, p}-u_{i, p} v_{p, i}\right) d V,
\end{gathered}
$$

it is seen that

$$
\begin{aligned}
D(u+v) & =\int_{V} C_{i j p q}\left(u_{i, j}+v_{i, j}\right)\left(u_{p, a}+v_{p, q}\right) d V \\
& =D(u)+D(v)+\int_{v} C_{i j p q}\left(u_{i, j} v_{p, a}+u_{p, a} v_{i, j}\right) d V \\
& =D(u)+D(v)+\int_{V}\left[C_{i, p q} u_{i, j} v_{p, a}+C_{p q i j} u_{i, j} v_{p, a}\right] d V \\
D(u+v) & =D(u)+2 D(u, v)+D(v) ;
\end{aligned}
$$

and

$$
\begin{gathered}
H(u+v)=H(u)+H(v)-\frac{1}{4} \int_{V} \sigma_{i j}\left[3\left(u_{p, i} v_{p, j}+v_{p, i} u_{p, j}\right)-\left(u_{i, p} v_{j, p}+v_{i, p} u_{i, p}\right)\right. \\
\left.-\left(u_{p, i} v_{j, p}+v_{p, i} u_{j, p}+u_{i, p} v_{p, j}+v_{i, p} u_{p, j}\right)\right] d V, \\
H(u+v)=H(u)+2 H(u, v)+H(v),
\end{gathered}
$$

since $\sigma_{i j}$ is symmetric.

The vector function $u_{i}=u_{i}\left(x_{1}, x_{2}, x_{3}\right)$ will be called an admissible vector if it is continuous and has continuous partial derivatives of at least the second order. The first eigenvalue of the differential equation (1) subject to boundary conditions (2) can then be found ${ }^{2}$ from the following minimum principle:

Of all admissible vectors $u_{i}$, that one which minimizes $D(u)$ under the side condition $H(u)=1$ is an eigenfunction of the differential equation (1) subject to boundary conditions (2). This minimum value of $D(u)$ is the corresponding eigenvalue $\lambda$.

It should be noted that this minimum principle can be rep aced by one requiring that $D(u) / H(u)$ be a minimum. The corresponding eigenfunction will then be determined only to within a factor of proportionality.

In order to prove this statement, let $u_{i}$ be the minimizing function and $\lambda$ the corresponding minimum value. Then

$$
D(u)=\lambda H(u) .
$$

Let $\xi_{i}$ be an arbitrary admissible vector and $\epsilon$ an arbitrary parameter. Then, since $u_{i}$ minimizes $D(u) / H(u)$, it follows that

$$
D(u+\epsilon \xi) \geqq \lambda H(u+\epsilon \xi) .
$$

${ }^{2}$ R. Courant and D. Hilbert, Methoden der mathematischen Physik, vol. 1, Julius Springer, Berlin, 1931, pp. 345-348. 
From Eqs. (9), (10), (11), this can be rewritten as

$$
2 \epsilon\left\{D(u, \xi)-\lambda H(u, \xi)+\frac{\epsilon}{2}[D(\xi)-\lambda H(\xi)]\right\} \geqq 0 .
$$

Since $\epsilon$ is chosen arbitrarily, the above relation will be valid only if

$$
D(u, \xi)-\lambda H(u, \xi)=0 .
$$

Now

$$
\begin{aligned}
D(u, \xi) & -\lambda H(u, \xi) \\
= & \int_{V}\left[C_{i j p d} u_{i, j} \xi_{p, a}+\frac{\lambda}{4} \sigma_{i j}\left(3 u_{p, i} \xi_{p, i}-u_{i, p} \xi_{i, p}-u_{p, i} \xi_{j, p}-u_{i, p} \xi_{p, i}\right)\right] d V \\
= & \int_{V}\left[C_{i j p q} u_{p, q} \xi_{i, j}+\frac{\lambda}{4} \sigma_{i j}\left(3 u_{p, i} \xi_{p, i}-u_{i, p} \xi_{j, p}-u_{p, i} \xi_{j, p}-u_{i, p} \xi_{p, i}\right)\right] d V=0 .
\end{aligned}
$$

By Green's theorem, this can be transformed into

$$
\begin{gathered}
\int_{S}\left\{C_{i i p q} u_{p, e} \xi_{i} n_{i}+\frac{\lambda}{4}\left[\sigma_{i j}\left(3 u_{p, i}-u_{i, p}\right) \xi_{p} n_{j}-\sigma_{i j}\left(u_{i, p}+u_{p, i}\right) \xi_{j} n_{p}\right]\right\} d S \\
-\int_{V}\left\{C_{i i p q} u_{p, a i} \xi_{i}+\frac{\lambda}{4}\left[\sigma_{i j}\left(3 u_{p, i}-u_{i, p}\right)\right]_{, j} \xi_{p}-\frac{\lambda}{4}\left[\sigma_{i i}\left(u_{i, p}+u_{p, i}\right)\right]_{, p} \xi_{j}\right\} d V,
\end{gathered}
$$

where $S$ is the bounding surface of $V$. By an appropriate interchange of subscripts, Eq. (12) can be written finally as

$$
\begin{aligned}
\int_{S} & {\left[C_{i j p q} u_{p, Q}+\frac{\lambda}{4} \sigma_{p i}\left(3 u_{i, p}-u_{p, i}\right)-\frac{\lambda}{4} \sigma_{p i}\left(u_{p, i}+u_{i, p}\right)\right] n_{j} \xi_{i} d S } \\
& -\int_{V}\left\{C_{i j p Q} u_{p, Q}+\frac{\lambda}{4}\left[\sigma_{p i}\left(3 u_{i, p}-u_{p, i}\right)-\sigma_{p i}\left(u_{p, i}+u_{i, p}\right)\right]\right\}, \xi_{i} d V=0 .
\end{aligned}
$$

Now the vector $\xi_{i}$ is chosen arbitrarily in the volume $V$ and on the boundary $S$; consequently

$$
\left\{C_{i, p Q} u_{p, q}+\frac{\lambda}{4}\left[\sigma_{p j}\left(3 u_{i, p}-u_{p, i}\right)-\sigma_{p i}\left(u_{p, i}+u_{j, p}\right)\right]\right\}_{, i}=0
$$

and

$$
\left\{C_{i, p q} u_{p, a}+\frac{\lambda}{4}\left[\sigma_{p j}\left(3 u_{i, p}-u_{p, i}\right)-\sigma_{p i}\left(u_{p, i}+u_{i, p}\right)\right]\right\} n_{i}=0 .
$$

These are identical with the differential equations (1) and boundary conditions (2).

Thus the solution of the minimum problem yields an eigenfunction and corresponding eigenvalue of the differential equation. It can also be seen that if an admissible function $v_{i}$ is used to generate an approximate solution, $D(v) / H(v) \geqq \lambda$, the actual eigenvalue.

3. Quasi-orthogonality and reality of the eigenfunctions. A quasi-orthogonality 
relationship between the eigenfunctions of Eq. (1), from which the reality of the eigenvalues follows, can be established by means of a standard technique. ${ }^{3}$ Define

$$
M_{i j}(u)=C_{i j p q} u_{p, a}
$$

and

$$
N_{i j}(u)=-\frac{1}{4}\left(3 \sigma_{i p} u_{i, p}-\sigma_{i p} u_{p, i}-\sigma_{i p} u_{i, p}-\sigma_{i p} u_{p, i}\right) .
$$

Then

$$
J_{i j}=M_{i i}(u)-\lambda N_{i j}(u) .
$$

Equation (1) takes the form

$$
\left[M_{i j}(u)-\lambda N_{i j}(u)\right]_{, i}=0,
$$

and boundary conditions (2) become

$$
\left[M_{i j}(u)-\lambda N_{i i}(u)\right] n_{i}=0 .
$$

Let $u_{i}$ and $v_{i}$ be two eigenfunctions and $\lambda$ and $\mu$ their respective eigenvalues, where $\lambda \neq \mu$. Then

$$
\left[M_{i j}(u)-\lambda N_{i j}(u)\right]_{, i}=0
$$

and

$$
\left[M_{i j}(v)-\mu N_{i j}(v)\right]_{, i}=0 .
$$

Multiplying the first by $v_{i}$ and the second by $u_{i}$, subtracting, and integrating yield

$$
\int_{V}\left[v_{i} J_{i, i}(u)-u_{j} J_{i, i}(v)\right] d V=0 .
$$

This can be rewritten by Green's theorem as

$$
\int_{S}\left[v_{i} J_{i j}(u)-u_{i} J_{i j}(v)\right] n_{i} d S-\int_{V}\left[v_{i, i} J_{i j}(u)-u_{i, i} J_{i j}(v)\right] d V=0 .
$$

But $J_{i j} n_{i}=0$ on $S$; therefore, the surface integral vanishes; also

$$
\begin{aligned}
\int_{V}\left[v_{i, i} M_{i j}(u)-u_{i, i} M_{i j}(v)\right] d V & =\int_{V}\left[v_{i, i} C_{i j p q} u_{p, q}-u_{i, i} C_{i j p a} v_{p, a}\right] d V \\
& =\int_{V} C_{i j p q}\left[v_{i, i} u_{p, q}-u_{p, a} v_{i, i}\right] d V=0 .
\end{aligned}
$$

Therefore, Eq. (15) becomes

$$
\lambda \int_{V} v_{i, i} N_{i j}(u) d V-\mu \int_{V} u_{i, i} N_{i j}(v) d V=0 .
$$

${ }^{8}$ L. Collatz, Eigenwertprobleme, Chelsea Publishing Co., New York, 1948, p. 61-65. 
Now

$$
\begin{aligned}
\int_{V} v_{i, i} N_{i i}(u) d V & =-\frac{1}{4} \int_{V} v_{i, i}\left(3 \sigma_{i p} u_{i, p}-\sigma_{i p} u_{p, i}-\sigma_{i p} u_{i, p}-\sigma_{i p} u_{p, i}\right) d V \\
& =-\frac{1}{4} \int_{V} u_{i, i}\left(3 \sigma_{p i} v_{i, p}-\sigma_{i p} v_{p, i}-\sigma_{i p} v_{i, p}-\sigma_{i p} v_{p, i}\right) d V \\
& =\int_{V} u_{i, i} N_{i j}(v) d V .
\end{aligned}
$$

Therefore,

$$
(\lambda-\mu) \int_{V} v_{i, i} N_{i j}(u) d V=0 .
$$

Since $\lambda \neq \mu$, it follows that

$$
\int_{V} v_{i, i} N_{i j}(u) d V=0
$$

Now let us consider

$$
\int_{V} v_{i}\left[M_{i j}(u)-\lambda N_{i j}(u)\right]_{, i} d V=0
$$

which can be recast as

$$
\int_{S} v_{j}\left[M_{i j}(u)-\lambda N_{i j}(u)\right] n_{i} d S-\int_{V} v_{i, i} M_{i j}(u) d V+\lambda \int_{V} v_{i, i} N_{i j}(u) d V=0 .
$$

But

$$
\left[M_{i j}(u)-\lambda N_{i j}(u)\right] n_{i}=0
$$

on $S$ and

$$
\int_{v} v_{j, i} N_{i j}(u) d V=0
$$

Therefore,

$$
\int_{V} v_{i, i} M_{i j}(u) d V=0
$$

Equations (16) and (17) are the quasi-orthogonality relations which hold between the eigenfunctions. It should be noted that these results are somewhat more complicated than the weighted orthogonality conditions usually found. This depends on the fact that the eigenvalues appear in the original differential equations and boundary conditions as coefficients of terms containing the highest order derivatives of the unknown functions.

Now assume that $\lambda$ is complex; that is, $\lambda=s+i t$ where $s$ and $t$ are real, and $t \neq 0$. Corresponding to $\lambda$ there will be a complex eigenfunction $u_{i}=\sigma_{i}+i \tau_{i}$ where $\sigma_{i}$ and $\tau_{j}$ are real vector functions and $\tau_{i} \not \equiv 0$. Since Eqs. (1) and (2) are linear with real coefficients, it follows that $\bar{\lambda}=s-i t$ is also an eigenvalue with corresponding eigen- 
function $\bar{u}_{i}=\sigma_{i}-i \tau_{j}$. A computation, similar to that used in the development of the quasi-orthogonality relation, will show that

$$
(\bar{\lambda}-\lambda) \int_{V} u_{i, i} N_{i j}(\bar{u}) d V=0 .
$$

Since $N_{i j}(u)$ is linear in the derivatives of $u_{i}$,

and

$$
N_{i j}(\bar{u})=N_{i j}(\sigma-i \tau)=N_{i j}(\sigma)-i N_{i j}(\tau)
$$

$$
\begin{aligned}
\int_{V}\left(\sigma_{i, i}+i \tau_{j, i}\right) N_{i j}(\sigma-i \tau) d V=\int_{V} & {\left[\sigma_{i, i} N_{i j}(\sigma)+\tau_{i, i} N_{i j}(\tau)\right] d V } \\
& \quad-i \int_{V}\left[\sigma_{i, i} N_{i j}(\tau)-\tau_{j, i} N_{i j}(\sigma)\right] d V .
\end{aligned}
$$

The imaginary part of this integral vanishes by the quasi-orthogonality relationship, and the integral reduces to

$$
\int_{V}\left[\sigma_{i, i} N_{i j}(\sigma)+\tau_{i, i} N_{i j}(\tau)\right] d V .
$$

If this integral does not vanish, then $\bar{\lambda}-\lambda=-2 i t=0$ and $\lambda$ is real. Thus the eigenvalues and eigenfunctions will be real if the integral appearing in (18) is non-zero.

Now

$$
\int_{V} \tau_{i, i} N_{i j}(\tau) d V=-\frac{1}{4} \int_{V} \sigma_{i j}\left(3 \tau_{p, i} \tau_{p, i}-\tau_{i, p} \tau_{p, i}-\tau_{p, i} \tau_{i, p}-\tau_{i, p} \tau_{j, p}\right) d V=H(\tau),
$$

and

$$
\int_{V} \sigma_{i, i} N_{i j}(\sigma) d V=H(\sigma)
$$

According to the minimum principle established in Section 2,

$$
\lambda=\min [D(u) / H(u)] .
$$

It has been assumed, tacitly, that a solution exists to this minimum problem. Under these conditions, $H(u)=0$ only if $D(u)=0$. But $D(u)$ is the strain energy of the perturbed state and will vanish only when the perturbation is a rigid body displacement. Consequently, $H(u) \neq 0$ if the minimum problem is to have a finite solution. Similarly, since $\sigma_{i}$ and $\tau_{i}$ are eigenfunctions of the differential equation (1), neither $H(\sigma)$ nor $H(\tau)$ can vanish if there is to be a finite, non-trivial solution. Integral (18) will then vanish only if $H(\sigma)$ and $H(\tau)$ are of opposite signs. If $H(u)$ is of one sign for all eigenfunctions, $u_{i}$, the eigenvalues and eigenfunctions must therefore be real. This restriction is not artificial, however, for $H(u)$ is the difference ${ }^{4}$ between the work of the surface stresses and the work of the body stresses $\lambda \sigma_{i j}$ on the strains associated with the displacements $u_{i}$. Thus if this work is always positive or always negative, the eigenvalues and eigenfunctions will be real. It is also seen that if $H(u)$ is of one sign for all admissible functions, the eigenvalue $\lambda$ will be of the same sign since $D(u) \geqq 0$. 